\title{
Characteristics of salivary profiles of oestradiol and progesterone in premenopausal women
}

\author{
Robert T Chatterton Jr ${ }^{1,2,5}$, Esnar T Mateo ${ }^{1}$, Nanjiang $\mathrm{Hou}^{4}$, \\ Alfred W Rademaker ${ }^{4}$, Simbi Acharya ${ }^{3}$, V Craig Jordan ${ }^{5}$ \\ and Monica Morrow ${ }^{3}$
} Departments of ${ }^{1}$ Obstetrics/Gynaecology, ${ }^{2}$ Physiology, ${ }^{3}$ Surgery, and ${ }^{4}$ Preventive Medicine, Robert H Lurie Comprehensive Cancer Center, ${ }^{5}$ Feinberg School
of Medicine, Northwestern University, Chicago, IL 60611 , USA
(Requests for offprints should be addressed to R T Chatterton, Department of Obstetrics/Gynaecology, Feinberg School of Medicine, Northwestern University,
710 N. Fairbanks Court, Chicago, IL 60611, USA; Email: chat@northwestern.edu)

\begin{abstract}
The objective of the study was to characterize salivary sex steroid levels in 56 women undergoing annual mammography who were participating in a breast density study at the Lynn Sage Breast Center of Northwestern Memorial Hospital, and to determine the predictability of the patterns within women. Saliva was collected daily by the women at home for one complete menstrual cycle and then again at approximately 6-month intervals. The occurrence of sporadic anovulatory cycles was identified in 12 subjects, and persistent oestradiol (OE2) elevation in all three cycles without significant progesterone levels occurred in another five subjects. In addition, both OE2 and progesterone were significantly lower in initial menstrual cycles than in subsequent cycles, suggestive of an
\end{abstract}

effect of participation in the study on hormone levels. Initial salivary OE2 levels were not good predictors of corresponding levels in either follicular or luteal phases of the menstrual cycles at the 6-month intervals. However, after the initial cycle, progesterone levels were highly predictable within individuals over a period of 6 months $(r=0.78, P<0.001)$. The study emphasizes the natural variation among and within women in the absence of any intervention, and indicates the need for properly controlled studies before attributing changes in hormonal levels to therapy. In addition, it emphasizes the importance of sampling at multiple time points when examining the relationship between hormones and risk.

Journal of Endocrinology (2005) 186, 77-84

\section{Introduction}

The success of endocrine therapy in improving survival in women with oestogen receptor positive breast cancer, coupled with the reduction in contralateral breast cancers seen with these agents (Early Breast Cancer Trialists' Collaborative Group 1998), has stimulated great interest in their use for chemoprevention. Tamoxifen reduces breast cancer incidence by $49 \%$ in high-risk women (Fisher et al. 1998). The availability of tamoxifen as an alternative to prophylactic mastectomy is a major advance, but side effects such as thrombophlebitis and an increased risk of endometrial cancer limit the use of tamoxifen. Investigations of new endocrine chemopreventive agents in premenopausal women necessitate monitoring of normal sex steroids to determine the impact of the drugs on the normal menstrual cycle and on fertility. This type of monitoring is important even for drugs not known to impact upon ovarian function.

Investigations into the endocrine effects of chemopreventive agents have been hampered by the need for multiple blood samples throughout the menstrual cycle. Saliva has a number of advantages over blood for the assessment of sex steroids over time: it can be easily collected by subjects in a painless fashion, it requires no special storage, and the steroid concentrations measured exclude the fraction which is bound to serum protein and biologically unavailable. Measurement of sex hormones in saliva provides a noninvasive means of assessing changes throughout the menstrual cycle, and allows reliable identification of the date of ovulation. We have adapted radioimmunoassys for the direct measurement of oestradiol (OE2) and progesterone in saliva, and have previously reported on the sensitivity, reliability, and correlations between serum and salivary OE2 and progesterone in these assays (Lu et al. 1997, 1999). We have also used saliva to examine intra- and inter-individual differences in sex hormone levels in premenopausal women (Gann et al. 2001). In a study of 12 women, Gann et al. (2001) compared cumulative and peak OE2 and progesterone concentrations for 2 consecutive menstrual cycles, and found a high within person correlation, although considerable 
variation among subjects was observed. Other studies have reported a low correlation between two luteal phase serum OE2 samples obtained over a 1 year interval, although sampling occurred approximately the same number of days after the onset of menses (Muti et al. 1996, Michaud et al. 1999). The aim of this study was to evaluate the variation in OE2 and progesterone levels in three menstrual cycles occurring during an 18-month period in a group of premenopausal women. Knowledge of the extent of the normal within-subject variation over a more prolonged time period is essential to accurately evaluate the effects of chemopreventive agents or other treatments in such women.

\section{Materials and Methods}

\section{Subjects}

Healthy women between the ages of 29 to 49 years were recruited from the Lynn Sage Breast Center of Northwestern Memorial Hospital after Institutional Review Board approval of the study. The study participants were women under the age of 45 who were undergoing annual mammography. Forty seven percent of the subjects had a family history of breast cancer. The study population was not meant to be representative of the general population, but rather of women attending breast clinics who would potentially participate in intervention studies. A medical history was obtained prior to entry into the study by face to face interview by a nurse, trained data manager, or physician. Weight and height were measured at presentation. Women were eligible if they were premenopausal with regular menses for the 6 months preceding study entry. Women were ineligible if they were taking oral contraceptives, tamoxifen, or other oestogen or progesterone containing drugs, were pregnant, lactating, planning pregnancy within the next year, or had been diagnosed with ovarian dysfunction. Use of oral contraceptives more than 6 months prior to study entry was permissible. Subjects who had a history of tamoxifen use for more than one month were ineligible.

Consenting subjects were given a box containing 32 vials with $0.5 \mathrm{mg}$ of $\mathrm{NaN}_{3}$ dried in the bottom as a preservative and sugarless chewing gum for saliva collection at the beginning of each collection cycle. Boxes with saliva samples were returned to the clinic by the subjects at the end of each monthly collection period. Collections were scheduled to be repeated every 6 months for three cycles. Beginning on the first day of menstrual bleeding, subjects began saliva collection. In the morning prior to food intake or tooth brushing, subjects chewed one-half a stick of sugarless gum to increase salivation and deposited $7-10 \mathrm{ml}$ of saliva into a vial, mixed the saliva with the preservative, and recorded the date of saliva collection on the vial and whether menstrual bleeding was present. This procedure was repeated daily for 30 days or until the beginning of the next menstrual period. Saliva was stored in a light-tight boxy in the subject's home without refrigeration and was brought to the Breast Center at the end of the month. Progesterone concentrations in samples prepared in this way are completely stable for at least 2 months (Lu et al. 1997, 1999). The stability of OE2 was tested again in this study by repeating assays of four samples that were stored at room temperature. No deterioration was observed in $\mathrm{OE} 2$ concentrations for four months.

\section{Samples and assays}

Upon receipt, samples were stored at $-20{ }^{\circ} \mathrm{C}$. For analysis the samples were thawed and centrifuged for $30 \mathrm{~min}$ at $5000 \mathrm{~g}$ to remove cellular debris. Batches of samples consisting of two month's collections were prepared to include two quality control preparations for estimating assay precision. The quality control preparation was a pool of saliva that had been stripped of steroids by incubation with agarose-coated charcoal and to which $44 \mathrm{pmol} / 1$ of OE2 and $636 \mathrm{pmol} / 1$ of progesterone had been added. OE2 and progesterone were assayed by direct radioimmunoassays as described in detail previously ( $\mathrm{Lu}$ et al. 1997, 1999). Briefly, the progesterone assay utilizes $\left[1,2,6,7-{ }^{3} \mathrm{H}\right]$ progesterone and an antiserum prepared by one of the authors ( $\mathrm{R} \mathrm{T} \mathrm{C}$ ) that has cross-reactivities of $0.5 \%$ with 17 -hydroxy $\mathrm{P}, 2.9 \%$ with pregnanedione, $0.95 \%$ with corticosterone, $11.4 \%$ with $5 \alpha$-pregnanedione, $1.9 \%$ with $5 \beta$-pregnanolone and $<0.1 \%$ with seven other steroids tested. Standards were prepared in $0.1 \mathrm{M}$ PBS, $\mathrm{pH} 7 \cdot 0$, containing $0 \cdot 015 \mathrm{M} \mathrm{NaN}_{3}$ and $0 \cdot 1 \%$ gelatin. The volume of sample used was $0 \cdot 1 \mathrm{ml}$. Intra-assay and interassay coefficients of variance (CV) were $7.9 \%$ and $19.9 \%$ respectively. Salivary OE2 was measured with a double antibody RIA, also described previously (Lu et al. 1999). Antiserum and ${ }^{125}$ I-labeled OE2 tracer were obtained from Diagnostic Systems Laboratories (Webster, TX, USA). The antiserum has cross-reactivities of $2 \cdot 4 \%$ with estrone, $0 \cdot 01 \%$ with estrone sulfate, $0 \cdot 21 \%$ with $16-$ ketooestradiol, $2 \cdot 6 \%$ with oestradiol-3-glucuride, $0 \cdot 64 \%$ with estriol, and $<0 \cdot 1 \%$ with non-phenolic steroids tested. The antiserum was diluted to give $40 \%$ binding. Standards were prepared by diluting a methanolic stock solution of OE2 with the same gelatin buffer used for progesterone. A precipitating antibody solution was prepared by titrating the amount of sheep antirabbit gamma globulin required for precipitation of $0.1 \mathrm{ml}$ rabbit serum, and adding this to propylene glycol $(4 \cdot 8 \mathrm{~g} / \mathrm{dl})$. The total volume of sample required was $0.4 \mathrm{ml}$. Intra- and interassay $\mathrm{CV}$ were $9 \cdot 9$ and $20 \cdot 2 \%$ respectively. Any samples with extremely high values (more than 3 S.D. above mean values from previous studies: $>1500 \mathrm{pmol} / \mathrm{L}$ for progesterone and $>100 \mathrm{pmol} / \mathrm{L}$ for estradiol) or with a pink or red color were tested for the presence of oxyhemoglobin with the 
Table 1 Characteristics of female subjects with hormone data in all three* sampling cycles

\begin{tabular}{|c|c|c|c|c|}
\hline & Total Group & $\begin{array}{l}\text { Women with complete } \\
\text { ovulatory cycles in all } \\
3 \text { cycles }\end{array}$ & $\begin{array}{l}\text { Women with anovulation } \\
\text { in } \geq \text { one cycle }\end{array}$ & $\begin{array}{l}\text { Women with high QE2 } \\
\text { in all } 3 \text { cycles }\end{array}$ \\
\hline Age (yr) & & & & \\
\hline Mean & $41 \cdot 9$ & $41 \cdot 1$ & $42 \cdot 9$ & $41 \cdot 6$ \\
\hline Median & $42 \cdot 0$ & $41 \cdot 0$ & $43 \cdot 5$ & $44 \cdot 0$ \\
\hline Range & $29 \cdot 0-49 \cdot 0$ & $34 \cdot 0-48 \cdot 0$ & $38 \cdot 0-46 \cdot 0$ & $29 \cdot 0-49 \cdot 0$ \\
\hline Median & $25 \cdot 8$ & $23 \cdot 8$ & $27 \cdot 3$ & $24 \cdot 2$ \\
\hline Range & $18 \cdot 4-43 \cdot 7$ & $20 \cdot 3-35 \cdot 5$ & $22 \cdot 2-38 \cdot 0$ & $10 \cdot 7-39 \cdot 4$ \\
\hline \multicolumn{5}{|c|}{ Gravidy $(\mathrm{G}) /$ Parity $(\mathrm{P})^{* *}$} \\
\hline $\mathrm{G}=0$ & $16(29 \%)$ & $7(33 \%)$ & $3(25 \%)$ & $2(40 \%)$ \\
\hline $\mathrm{G}>0, \mathrm{P}=0$ & $7(13 \%)$ & $3(14 \%)$ & $2(17 \%)$ & $1(20 \%)$ \\
\hline $\mathrm{G}>0, \mathrm{P}>0$ & $32(58 \%)$ & $11(53 \%)$ & $7(58 \%)$ & $2(40 \%)$ \\
\hline
\end{tabular}

*Sixteen women missed one or more of the three cycles, and are not included in this table. ${ }^{* *}$ Number of subjects with percentages of the group in parentheses. OE2, oestradiol.

Hemoccult procedure (Beckman-Coulter, Fullerton, CA, USA). No positive tests were found.

\section{Analyses}

The last day of the menstrual cycle (the day before menstrual bleeding) was designated cycle-day zero. Saliva samples from days -28 through the end of the cycle were assayed for OE2. Those from days -17 through day-zero were assayed for progesterone. The criteria used to determine the day of ovulation were: 1) progesterone values of $>190 \mathrm{pmol} / 1$ or more on 3 or more days in a 4-day period occurring within days -4 to -10 from the end of the cycle. This was an arbitrary value based upon a detectable increase over follicular phase levels based on a previous study of similar, ovulatory subjects in whom conception had occurred (Vitzthum et al. 2002); 2) cycle-length of $>24$ days, and 3) OE2 values of $<44 \mathrm{pmol} / 1$ during the first 10 days of the cycle. With these criteria met, the day of ovulation was determined by counting back 13 days from the last day of the cycle. Usually, there was a peak of $\mathrm{OE} 2$ within \pm 2 days from day -13 . If so, we choose the day after the peak as the day of ovulation (Lipson \& Ellison 1996). For purposes of displaying the data the cycle was renumbered with the day of ovulation assigned as day zero. The follicular phase was numbered negatively and luteal phase positively from the day of ovulation (day zero was included with the follicular phase for this comparison). Comparisons among and within subjects were made using average values for the follicular and luteal phases.

Mean age and BMI were compared across groups defined by ovulatory status using one-way analysis of variance. Parity was compared across these groups using Fisher's exact test. Untransformed mean hormone levels are presented by cycle for the luteal and follicular phases.
Within each phase, differences in mean levels are compared between cycles using the paired t-test. Mean hormone levels were compared between groups using the independent sample $t$-test. Data were log-transformed for these statistical tests. Outliers were assessed using the generalized extreme studentized deviate method of Rosner (1983), and their frequency and influence was reduced by log-transforming. Intra-class correlations (ICCs) and their confidence intervals were calculated using between-woman and within-woman variance components from a random effects model analysis of the log-transformed data (Rosner 2000). Spearman correlations were calculated between hormone levels at different visits.

\section{Results}

Fifty-six women with a median age of 42 years entered the study. Patient characteristics are summarized in Table 1 for the 38 women who had hormone data at all three cycles. Fifty-four of the 56 women had at least one ovulatory cycle including 21 in which all 3 cycles were collected and were ovulatory. Twelve subjects also provided samples for all three cycles, but 13 cycles in these subjects were classified as anovulatory. An additional five subjects were anovulatory in all three cycles, had high levels of OE2, and were analyzed separately. Sixteen subjects did not complete collections of saliva in one or more months. The mean interval between the first and second sampling periods was $215 \pm 55$ ( \pm S.D.) days and that between the second and third periods was $227 \pm 72$ ( \pm S.D.) days. The age, body mass index (BMI) and reproductive histories of women in these three groups are compared in Table 1. The median age of the entire group was 42 years, the median BMI was 


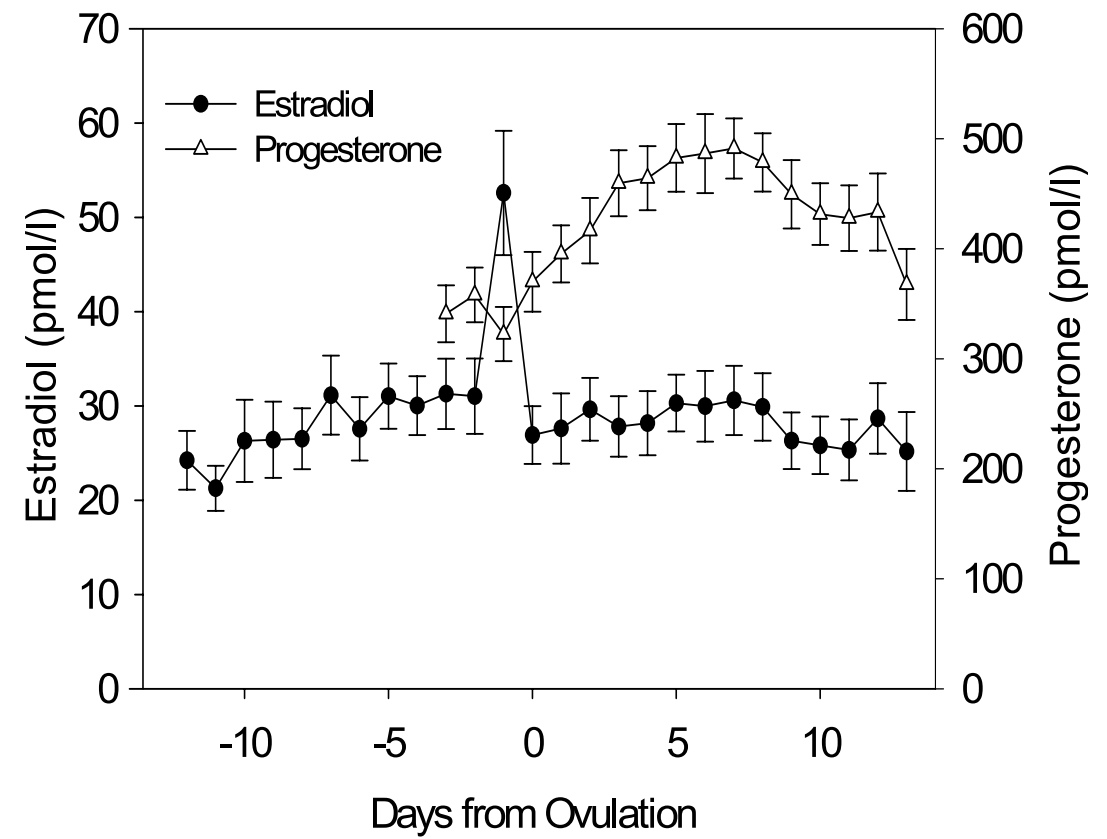

Figure 1 Concentrations of oestradiol and progesterone in saliva throughout the menstrual cycle in 110 cycles in 54 ovulatory women. The estimated day of ovulation from criteria described in Materials and Methods is shown as day zero.

$25 \cdot 8$, and $58 \%$ of the subjects were parous. No significant differences in age $(P=0.45)$, BMI $(P=0.52)$, or parity $(P=0.95)$ were observed among three groups on the basis of ovulatory status. The mean values of OE2 and progesterone for each cycle day with their standard errors are shown in Fig. 1 for all 110 ovulatory cycles in 54 subjects. Subjects with a single anovulatory cycle were eliminated from the analyses of trends among cycles. Among ovulatory cycles, the percentage of missed days (no saliva) averaged $2.9 \%$ in the first cycle, $0.8 \%$ in the second, and $2 \cdot 8 \%$ in the third. In the 21 subjects with three ovulatory cycles the mean OE2 level in the follicular phase across all three cycles was $22 \cdot 1 \pm 2 \cdot 7$ ( \pm S.E.) pmol/l. Across the luteal phase the mean OE2 was $20 \cdot 6 \pm 2 \cdot 4$ ( \pm S.E.) pmol/1 while mean luteal progesterone was $436 \pm 34$ ( \pm S.E.) pmol/1. The follicular phase of the first cycle had lower levels of OE2 than the third cycles $(P=0 \cdot 01$, Table 2$)$. In the luteal phase the levels of progesterone of the first cycle were significantly lower than in cycle $2(P=0 \cdot 004)$ and the levels of OE2 of the first cycle were significantly lower than those of cycle $3(P=0 \cdot 015$, Table 2$)$. The decreased levels of OE2 and progesterone in cycle 1 were not explained by interassay variation over time. There was no drift in the concentration of the quality control (QC) preparation that was assayed with each batch over the course of the study. The plots of QC values with time were linear for both OE2 and progesterone. For OE2 the slope was $0.067 \pm 0.057 \mathrm{pg} / \mathrm{month}$ and for progesterone the slope was $-0.072 \pm 0.048 \mathrm{pg} / \mathrm{month}$. Also, only $10.5 \%$ of first cycles had been assayed before the first of the

Table 2 Concentrations of salivary oestradiol (OE2) and progesterone $(\mathrm{P})$ in premenopausal women (mean \pm s.E.). Menstrual cycles were separated by a mean of $7 \cdot 2$ months

\begin{tabular}{|c|c|c|c|}
\hline Menstrual cycle & $\begin{array}{l}\text { Oestradiol } \\
(\mathrm{pmol} / \mathrm{l})\end{array}$ & $\begin{array}{l}\text { Progesterone } \\
(\mathrm{pmol} / \mathrm{l})\end{array}$ & $\begin{array}{l}P \text { value } \\
\text { between cycles }\end{array}$ \\
\hline \multicolumn{4}{|l|}{ Follicular phase } \\
\hline Cycle 1 & $13 \cdot 0 \pm 1 \cdot 9$ & - & $0 \cdot 01,1$ vs 3 \\
\hline Cycle 2 & $25 \cdot 2 \pm 5 \cdot 8$ & - & - \\
\hline Cycle 3 & $28 \cdot 1 \pm 5 \cdot 1$ & - & - \\
\hline \multicolumn{4}{|l|}{ Luteal phase } \\
\hline Cycle 1 & $13 \cdot 1 \pm 2 \cdot 4$ & $329 \pm 39$ & $0 \cdot 004,1$ vs 2 , P only \\
\hline Cycle 2 & $22 \cdot 3 \pm 4 \cdot 8$ & $517 \pm 43$ & - \\
\hline Cycle 3 & $26 \cdot 3 \pm 4 \cdot 4$ & $462 \pm 81$ & $0 \cdot 015,1$ vs 3 OE2 only \\
\hline
\end{tabular}




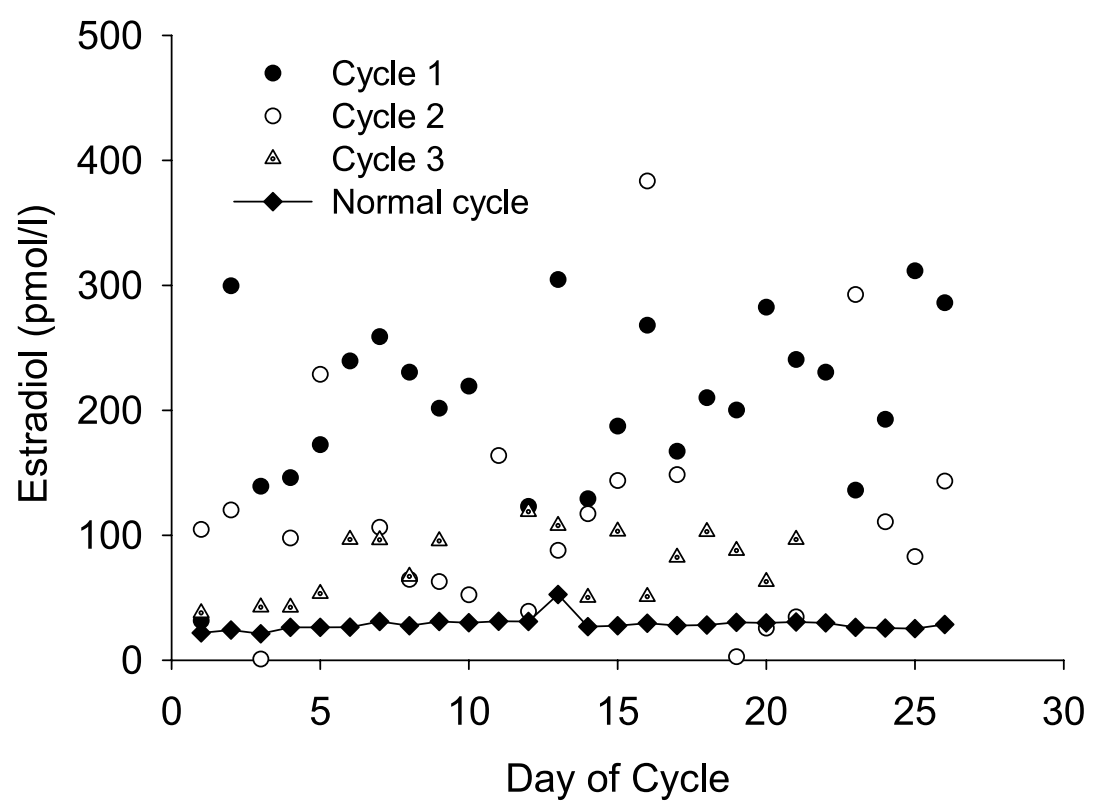

Figure 2 Salivary oestradiol in a subject with consistently elevated oestradiol concentrations. The levels in 110 cycles in 54 ovulatory subjects are shown in the same scale (normal cycle). Because the day of ovulation cannot be ascertained in cycles with persistently high estradiol levels, the cycle is dated forward from the first day of menstrual bleeding.

second cycles was assayed: $7 \%$ of second cycles were assayed after all first cycles had been assayed. A similar overlap occurred between the second and third cycles.

For those cycles designated as anovulatory (13 cycles in 12 different women) the OE2 level was $28 \cdot 1 \pm 7 \cdot 9$ ( \pm S.E.) $\mathrm{pg} / \mathrm{ml}$ and the progesterone level was $89 \cdot 0 \pm 16 \cdot 1$ ( \pm S.E. $)$ $\mathrm{pg} / \mathrm{ml}$. The levels of OE2 in ovulatory subjects (follicular phase) and anovulatory subjects were not significantly different $(P=0.82)$, but that for progesterone was less $(P<0 \cdot 0001)$. The mean OE2 for the five subjects with high levels of E2 was $77 \cdot 5 \pm 10 \cdot 4$ ( \pm S.E.) $\mathrm{pmol} / 1$ and that for progesterone was $69 \cdot 3 \pm 25 \cdot 5$ ( \pm S.E.) $\mathrm{pmol} / \mathrm{l}$. The OE2 concentration is significantly greater $(P<0.0001)$ and the progesterone is significantly less $(P=0 \cdot 001)$ than that of the ovulatory subjects. The pattern of OE2 throughout the cycle for the three menstrual cycles is shown for one of these five subjects (Fig 2).

The mean level of the hormones in the follicular phase of ovulatory subjects was compared among the cycles within subjects. The Spearman correlation between first and second visits and between second and third visits was determined. Similar comparisons were made for luteal phases. There was no significant correlation between OE2 levels across visits in either follicular or luteal phases. For progesterone there was a significant correlation in luteal phases between cycles 2 and 3, where the correlation was $0.78(P<0.001)$ and the ICC was $0.64(95 \%$ C.I. 0.29 to $0 \cdot 83$ ). The ICC did not differ by age or BMI.
Overall, $47 \%$ of the subjects had a family history of breast cancer. Four of the five subjects with high levels of OE2 had a family history of breast cancer.

\section{Discussion}

The levels of OE2 and progesterone observed in this study are similar to those reported in studies in which the method was validated (Lu et al. 1997, 1999) and are similar to, but somewhat higher, than those previously reported by the Harvard group (Lipson \& Ellison 1996, Furberg et al. 2005). In all studies a distinct preovulatory peak is evident. The lower levels of OE2 in the first half of the follicular phase are balanced by inclusion of the preovulatory peak of OE2 in the follicular phase. Nevertheless, the pattern of OE2 in saliva during the cycle is less variable than that commonly observed in serum. This presumably relates to the fact that salivary OE2 represents the 'available' fraction of OE2 in blood, i.e. the fraction not bound to sex hormone-binding globulin (Plymate et al. 1990). Other investigators have published data on salivary progesterone and OE2 levels during the menstrual cycle (Evans et al. 1980, Choe et al. 1983, O’Rourke \& Ellison 1993, Lipson \& Ellison 1996) but this study is the first to examine the consistency of patterns within subjects over a period of more than a year.

We previously studied the reproducibility of OE2 and progesterone levels in consecutive menstrual cycles of 
normal ovulatory women, and compared the reproducibility of the salivary hormones to the sex steroids measured in blood samples from the same cycles (Gann et al. 2001). The reproducibility of OE2 within subjects was high; the variance within subjects was $<1 / 6$ of that between subjects (ICC of 0.86) and reproducibility was higher than that of timed blood samples. Progesterone reproducibility was also high; the variance within subjects was $1 / 3$ of that between subjects (ICC of 0.75 ). In the present study the average time interval between studied menstrual cycles was $7 \cdot 5$ months. For OE2 there was no correlation within subjects in follicular or luteal phases of the three cycles, and the ICC ratios showed that variance within subjects over time was greater than that between subjects. It is not surprising that cycles separated by over 7 months would not be correlated. However, although salivary progesterone also had no correlation between cycle 1 and cycle 2, it was surprisingly highly correlated between cycles 2 and 3 within subjects $(r=0.78$ and ICC of 0.64$)$. This is similar to the relationship between consecutive cycles. The reason that progesterone of the first cycle in this study did not correlate with the other cycles is a matter for speculation. Other than the difference in time between cycles, there was a difference in the subject population; subjects in the present study were women undergoing annual mammography, many of whom were at increased risk for breast cancer development, while those in the earlier study (Gann et al. 2001) were a convenience sample recruited by newspaper announcements.

In addition to the lack of correlation of progesterone levels between the first cycle and the other cycles, the progesterone concentrations in the initial cycle were significantly lower. It is possible that entry into the study under these conditions had a suppressive effect on ovarian function that affected individuals differently. A psychological effect on ovarian function is not unknown (Chatterton 1990). It will be interesting to ascertain whether the psychological aspects of entering such a study are, in fact, the cause of the suppression of salivary progesterone in such subjects. The physical stress of exhausting aerobic activity has been shown to decrease salivary progesterone in women (Ellison \& Lager 1986) but the effect of psychological stressors on salivary hormone levels of sex steroids has not been specifically investigated. Why progesterone would be more consistent than OE2 within women over periods of 6 months is not evident from the data. However, menstrual cycle length will affect mean values of OE2 in the follicular phase more than mean values in the luteal phase which is more stable in length. Also, the greater coefficient of variation in OE2 values will affect the ability to detect a correlation.

It is of interest to compare OE2 levels in ovulatory women in this higher risk cohort vs the convenience (Gann et al. 2001) groups. The mean OE2 levels across cycle days -6 to +8 for the high risk and convenience groups were $460 \pm 53( \pm$ S.D. $)$ and $507 \pm 71$ ( \pm S.D. $)$ $\mathrm{pmol} / 1$ respectively $(P=0.52)$. Thus, in this small sample there is no evidence for a higher level of OE2 in the ovulatory, high risk women.

Anovulatory cycles were found only sporadically. No individual had anovulatory cycles in all three cycles. The OE2 level in anovulatory cycles was not less than that of the follicular phases of ovulatory cycles. This is an unexpected finding and, if salivary OE2 were to be used for assessment of ovulatory dysfunction, this observation should be confirmed by additional study. However, since none of these subjects had chronic anovulation, aspects of characteristic pituitary or hypothalamic dysfunction may not apply.

The five women with chronic elevation of OE2 and absence of a mid-cycle elevation in salivary progesterone are interesting. They may have a variation of polycystic ovary syndrome (PCOS), although this was not diagnosed as such prior to the study. Women with PCOS generally have total serum OE2 levels that are within the normal range; however, OE2 concentrations that are not bound to sex steroid-binding globulin (SHBG) have been shown to be, on average, more than twice as high in PCOS than in normal control subjects (Lobo et al. 1981). Blood was drawn from the normal women in that study between days 2-7 of the menstrual cycle. The reason for the elevation in serum SHBG-unbound OE2 is likely because serum SHBG concentrations are suppressed in the presence of the elevated androgen levels (Edmunds et al. 1990) that are characteristic of PCOS. The higher levels of serum SHBG-unbound OE2 would result in greater transfer of OE2 to tissues including salivary glands. Alternatively, cases of peripheral aromatase overexpression through gainof-function mutations have been described (Shozu et al. 2003). A diagnosis of these patients has not yet been established. However, monitoring of salivary levels may prove to be a useful tool for monitoring response to therapy in women with ovarian dysfunction. It is interesting that four of the five women with high levels of OE2 had a family history of breast cancer. However, this is a very small sample and previous studies do not uniformly support a relationship between PCO and breast cancer (Atiomo et al. 2003, Pierpoint et al. 1998, Anderson et al. 1997).

The availability of this noninvasive procedure makes monitoring the effects of chemoprevention agents on sex steroid levels feasible. Saliva samples can be collected at home by the patients and stored without refrigeration until brought to the laboratory at the end of the month. The pattern of hormones for evaluation of complete menstrual cycles can thus be obtained painlessly and inexpensively by this procedure. Although the need for monitoring of endocrine chemopreventive agents is obvious, monitoring of other agents may identify unexpected effects on fertility or ovarian function. The epidermal growth factor receptor inhibitors are currently being evaluated as breast cancer therapeutic, and possibly preventive agents (Lu et al. 2003). 
However, recent work indicates that luteinizing hormone $(\mathrm{LH})$ induces expression of epidermal growth factor family members which mediate the effect of LH action on the ovulatory follicle (Park et al. 2004), indicating that monitoring of the reproductive effects of these agents could provide valuable information.

In conclusion, it is evident that daily monitoring of salivary sex steroids can provide important information on infrequent and subtle but significant alterations in availability of hormones to their target organs. In this group of 56 women undergoing annual mammography and reporting regular menstrual cycles prior to study entry we have identified the occurrence of sporadic anovulatory cycles in 12 subjects and persistent OE2 elevation in another five subjects. In addition, evidence that initiation of this kind of a study may have suppressive effects on salivary OE2 and progesterone levels was observed. Salivary OE2 levels were not predictive of levels in either follicular or luteal phases of future menstrual cycles after average periods of $7 \cdot 5$ months. However, after the initial cycle, progesterone levels were highly predictable within individuals. The study emphasizes the natural variation among and within women in the absence of any intervention, and indicates the need for properly controlled studies before attributing changes in hormonal levels to therapy. In addition, it emphasizes the importance of sampling at multiple time points when examining the relationship between hormones and risk.

\section{Acknowledgments}

Supported by a grant from the USPHS, NIH, NCI, P50 CA89018-01 and the Avon Foundation. None of the authors have any conflict of interest that would prejudice the impartiality of the manuscript.

\section{References}

Anderson KE, Sellers TA, Chen PL, Rich SS, Hong CP \& Folsom AR 1997 Association of Stein-Leventhal syndrome with the incidence of postmenopausal breast carcinoma in a large prospective study of women in Iowa. Cancer 79 494-499.

Atiomo WU, El-Mahdi E \& Hardiman P 2003 Familial associations in women with polycystic ovary syndrome. Fertility and Sterility $\mathbf{8 0}$ 143-145.

Chatterton RT Jr 1990 The role of stress in female reproduction: animal and human considerations. International Journal of Fertility 35 $8-13$.

Choe JK, Khan-Dawood FS \& Dawood MY 1983 Progesterone and estradiol in saliva and plasma during the menstrual cycle. American Journal of Obstetrics and Gynecology 147 557-562.

Early Breast Cancer Trialists' Collaborative Group 1998 Tamoxifen for prevention of breast cancer: An overview of the randomized trials. Lancet 351 1451-1467.

Edmunds SEJ, Stubbs AP, Santos AA \& Wilkinson ML 1990 Estrogen and androgen regulation of sex hormone binding globulin secretion by a human liver cell line. Journal of Steroid Biochemistry and Molecular Biology 37 733-739.
Ellison PT \& Lager C 1986 Moderate recreational running is associated with lowered salivary progesterone profiles in women. American Journal of Obstetrics and Gynecology 154 1000-1003.

Evans JJ, Stewart CR \& Merrick AY 1980 Oestradiol in saliva during the menstrual cycle. British Journal of Obstetrics and Gynecology 87 624-626.

Fisher B, Costantino JP, Wickerham DL, Redmond CK, Kavanah M, Cronin WM, Vogel V, Robidoux A, Dimitrov N, Atkins J, Daly M, Wieand S, Tan-Chiu E, Ford L \& Wolmark N 1998

Tamoxifen for prevention of breast cancer: Report of the National Surgical Adjuvant Breast and Bowel Project P-1 Study. Journal of the National Cancer Institute 90 1371-1388.

Furberg A-S, Jasienska G, Bjurstam N, Torjesen PA, Emaus A, Lipson SF, Ellison PT \& Thune I 2005 Metabolic and hormonal profiles: HDL cholesterol as a plausible biomarker of breast cancer risk. The Norwegian EBBA study. Cancer Epidemiology, Biomarkers and Prevention 14 33-40.

Gann PH, Giovanazzi S, Van Horn L, Branning A \& Chatterton RT Jr 2001 Saliva as a medium for investigating intra- and interindividual differences in sex hormone levels in premenopausal women. Cancer Epidemiology, Biomarkers and Prevention 10 59-64.

Lipson SF \& Ellison PT 1996 Comparison of salivary steroid profiles in naturally occurring conception and non-conception cycles. Human Reproduction 11 2090-2096.

Lobo RA, Granger L, Goebelsmann U \& Mishell DR Jr 1981 Elevations in unbound serum estradiol as a possible mechanism for inappropriate gonadotropin secretion in women with PCO. Journal of Clinical Endocrinology and Metabolism 52 156-158.

Lu Y-C, Chatterton RT Jr, Vogelsong KM \& May LK 1997 Direct radioimmunoassay of progesterone in saliva. Journal of Immunoassay 18 149-163.

Lu Y-C, Bentley GR, Gann PH, Hodges KR \& Chatterton RT Jr 1999 Salivary estradiol and progesterone levels in conception and non-conception cycles in women. Evaluation of a new assay for salivary estradiol. Fertility and Sterility 71 863-868.

Lu C, Speers C, Zhang Y, Xu X, Hill J, Steinbis E, Celestino J, Shen Q, Kim H, Hilsenbeck S, Mohsin SK, Wakeling A, Osborne CK \& Brown PH 2003 Effect of epidermal growth factor receptor inhibitor on development of estrogen receptor-negative mammary tumors. Journal of the National Cancer Institute 95 1825-1833.

Michaud DS, Manson JE, Spiegelman D, Barbieri RL, Sepkovic DW, Bradlow HL \& Hankinson SE 1999 Reproducibility of plasma and urinary sex hormone levels in premenopausal women over a one-year period. Cancer Epidemiology, Biomarkers and Prevention 8 1059-1064.

Muti P, Trevisan M, Micheli A, Krogh V, Bolelli G, Sciajno R \& Berrino F 1996 Reliability of serum hormones in premenopausal and postmenopausal women over a one-year period. Cancer Epidemiology, Biomarkers and Prevention 5 917-922.

O’Rourke MT \& Ellison PT 1993 Salivary estradiol levels decrease with age in healthy, regularly-cycling women. Endocrine Journal 1 487-494.

Park JY, Su YQ, Ariga M, Law E, Jin SL \& Conti M 2004 EGF-like growth factors as mediators of $\mathrm{LH}$ action in the ovulatory follicle. Science 303 682-684.

Pierpoint T, McKeigue PM, Isaacs AJ, Wild SH \& Jacobs HS 1998 Mortality of women with polycystic ovary syndrome at long-term follow-up. Journal of Clinical Epidemiology 51 581-586.

Plymate SR, Namkung PC, Metej LA \& Petra PH 1990 Direct effect of plasma sex hormone binding globulin (SHBG) on the metabolic clearance rate of $17 \beta$-estradiol in the primate. Journal of Steroid Biochemistry 36 311-317.

Rosner B 1983 Percentage points for a generalized ESD many-outlier procedure. Technometrics 25 165-172.

Rosner B 2000 Fundamentals of Biostatistics, Chapter 12, Multisample inference, pp 511-576. Pacific Grove, CA: Duxbury Press. 
Shozu M, Sebastian S, Takayama K, Hsu WT, Schultz RA, Neely K, Bryant M \& Bulun SE 2003 Estrogen excess associated with novel gain-of-function mutations affecting the aromatase gene. New England Journal of Medicine 348 1855-1865.

Vitzthum VJ, Bentley GR, Spielvogel H, Caceres E, Thornburg J, Jones L, Shore S, Hodges KR \& Chatterton RT 2002 Salivary progesterone levels and rate of ovulation are significantly lower in poorer than better-off urban-dwelling Bolivian women. Human Reproduction 17 1906-2002.

Received in final form 16 February 2005 Accepted 29 March 2005 